\title{
A new approach in Supply Chain Processes - Smart Logistics
}

\author{
Olga Bucovetchi ${ }^{1,{ }^{*}}$, Andreia Elena Simioana ${ }^{1}$, and Radu D. Stanciu ${ }^{1}$ \\ ${ }^{1}$ University POLITEHNICA of Bucharest, 313 Spl. Independentei, 060042 Bucharest, Romania
}

\begin{abstract}
Globalization is progressing steadily and cannot be stopped. Through existing technologies, this process will be accelerated further. Entire professions and industries, as well as government and politics, must constantly adapt to this process. Many things can be created quickly, which, in turn, creates more vulnerability. These changes are supported by different technologies, which also change rapidly, not to mention the perception of time influencing people's awareness. Things are faster and sometimes more fragile. In this context, supply chain is perceived as a mean of expressing globalization. The present paper emphasizes the switch of paradigm from classic logistics to smart logistics. The authors analysed several prestigious reports (released by World Economic Forum, Oxford Martin Commission for Future Generations, IEEE Computer Society, etc.) to highlight the global trends in supply chain processes. The main objective of the research is to shape the near future of logistics to prepare the production and transportation industry for change.
\end{abstract}

\section{Introduction}

Globalization means transforming the economy, culture, innovation and trade in new global synthesis, a new debate about how our civilization could develop into something better. Globalization refers to the close collaboration between nations in a free trade world, sharing global concerns such as climate, energy, economy, technology, security and democracy. Globalization will support several new advanced systems. Industries around the world are working together to find better ways of doing business. The main objective of the research is to shape the near future of logistics to prepare the production and transportation industry for change.

The paper presents, in great, a literature research towards mega trends revealed by prestigious worldwide entities. This study highlights mostly theoretical things about the future of globalization regarding Supply Chain Management.

The literature research shall represent the starting point for a deeper analysis in order to help practitioners to reshape their businesses, to embed these trends within the organizational behaviour to be more globally competitive.

\footnotetext{
*Corresponding author: olga.bucovetchi@upb.ro
} 


\section{Research methodology}

After comprehensive trend analyses, a wide-ranging literature review was conducted and summarized [1-4]. Existing and new concepts, techniques to meet current challenges were investigated, analysed and positioned. Essentials have been identified and priorities were set as well and findings have been summarized and analysed.

It is important to note several aspects of risk concepts generated by these reports. First, updates, maintenance, and having a consistent implementation are difficult to carry out. The relationships between risks are also difficult to recognize and maintain, especially when they are difficult or impossible to understand. Right now, the global current risks maps are the most comprehensive and concrete, and have the most sustainable overviews, but these also examine risk at a global level, and not necessarily at smaller levels, where the risk(s) may have more significant impacts. Over the years, risk has shifted and some dependencies have now been documented and are visible. Some of these risks have been quantified and documentation is available to help mitigate these risks as they occur. There is a communal consensus that global risk assessment is an important instrument to promote risk sensitivity and awareness. However, the responsibility as to who is responsible for identifying, dealing with, or communicating risk factors is often unclear and inconsistent, and the consequences are not fully outlined. It is important to note that there is a lot of know-how and new technologies that are required to master risks as they occur. The dynamics and collaboration of risk management are hard to visualize and the different ideas of quality affinity are a challenge. Many resources are required for risk mitigation and can only, unfortunately, be provided by just a few. The related yet different assessments of countries, regions, or cultures are not documented. Because implementation is not always consistent, new risks can be created. It is also always important to note that people are still unpredictable and it is difficult to have risk that is manageable.

\section{Main findings}

The following trends provided by research institutes and globally focused organizations are currently leading the way. These have shaped the development of new transport management systems to gain a higher market share in logistics area.

The risk landscape developed and published by the World Economic Forum (WEF) was used as a source for the research because it includes over 10 years of documented global risks and are regularly updated by an accepted body. This risk landscape is very comprehensive, as the perception of risk over time changes and differences are perceived and implemented

Many companies compete through storage and transport services provided to meet a growing number of online orders, which is today the main driver of logistics. As a result, received more orders and a greater diversity ordered naturally requires a larger deposit. Fortunately, some carriers have a plan to improve warehouse utilization. The easiest way to make a deposit effectively is through a proper inventory management, but it just means more efficient stored products, and products not delivered.

To honour the high volume of orders and to have satisfied customers, retailers must embrace new technological trends and to understand the current state of logistics in electronic commerce to prepare for the future needs of commerce. [5] They must invest in devices that eases the work and soon in robotics, Amazon changing landscape in the supply chain and delivery robotics.

Transport industry is going through some remarkable changes now; these changes are leading up to a certain long-term impact that will change goods' movements in the world. 
Transportation management systems (TMS) will be centralized in Cloud. TMS solutions must work with all aspects of transport, from inventory management to status updates of carriers in transit. For any TMS, one of the main tasks is to gather real-time information from all parties involved in the transport cycle. Given this, visibility becomes one of the most crucial factors in increasing the efficiency of TMS solutions, thus information gathering becomes even more important.

Transportation Management is headed by Internet of Things (IoT), which supports carriers by offering ways to retrieve information, self-service and automatic updating through portals. Thus, companies can access information from any location and take advantage of the latest information from all partners in the supply chain network. This TMS solutions help generate higher output in terms of efficiency and reduction in operational costs.

Logistics flows, payments and transactions will need to satisfy requirements related to the enormous trade economy based on innovations. Many of these chains will be automated and will work online to receive orders from one end of the world, to honour others at the other end and produce elsewhere. These transactions will be further accelerated by the visibility offered by internet, wireless connection in real time and a new digital global financial standard.

Within the book "New Mega Trends" [6], Frost \& Sullivan expert, Sarwant Singh claims that "SMART is the new green". Furthermore, he predicts "the failure of green technology to show a manageable and predictable return on investment that will lead to a shift towards intelligent products, services and even cities that 'behave' green by adapting and changing to the environment around them. The megatrend of the last decade - Green products - will be replaced in this decade by Smart products and services". A smart product is characterized by an intelligent sensing technology that is increasingly being integrated with Internet technologies, thereby allowing the product to react to and communicate with the changing environment around it. This leads to optimal operations and improvement in terms of efficiency.

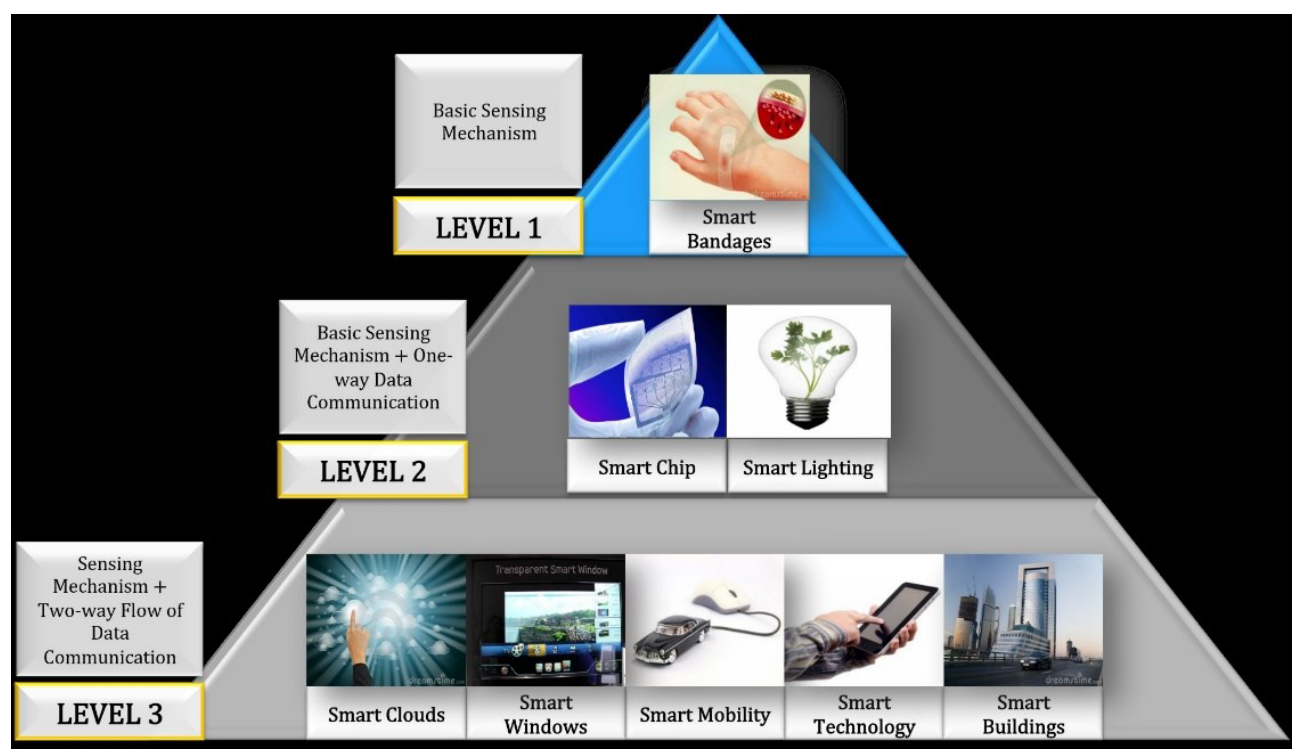

Fig. 1. SMART products, [7]

As one may observe from figure 1, there is a large number of SMART products, which can be structured in 3 levels, depending on their connectivity, from basic sensing 
mechanism to two-way flow of data communication. If analysing the first two levels, one may highlight the time gap between the sensor signing the problem or the misbehaviour of an apparatus and the moment of parameters' recovery, while on the thirds level of technology, one can speak about autonomous decision-making process - technology recovering by itself.

Currently logistics is controlled by simple transport processes; modern logistics will be composed of data, this concept will become essential for success, and the Internet started to become mountaintop informational data, which with the help of IT systems are analysed for insights and better transport operations. Every device that is connected to Internet via Wi-Fi or a wired connection generates more information for enterprises and carriers that are used in real-time by them. Meanwhile, technology is increasing to meet demand and current pressures on the industry

Virtual reality will be used in coming years in storage services; if nowadays scanning is done using mobile packages, in the coming years will be done with the help of glasses which visually scan all packages. On average, a person can hold approximately seven bits of information in short-term memory, but with these glasses and the data on parcels will immediately scanned and sent to a database. As a consequence, the working time of human workforce will be reduced, the inventory will be done automatically, this providing instantly updated information about the free space in storage.

Recently, global attention was directed toward unmanned aerial vehicles, (UAV), which are already used for military purposes in Pakistan (US Army) for operating aid in the development of Africa and delivery packages in Germany (DHL Packet).

Besides military purposes, the world's leading online retailer Amazon and the largest logistics company in the world, DHL have already started using these devices for delivering parcels.

Using drones in logistics represents a double-edged sword even if they have the advantage of a reduced delivery-time: on the one hand because of the cost of delivering very high, and on the other hand in terms of the number of devices that would be needed to cover the need of delivery. Moreover, it's a matter of concern when taking into consideration the air safety. Airspace would very much clutter, and the smallest error on these devices could have dramatic effects and can even shoot down a plane full of passengers.

To avoid such incidents, large companies increasingly invest in testing several types of unmanned aerial vehicles, as those organizations are confident that these devices will be the future of efficient logistics

\section{Conclusions}

Supply chain industry leaders have access to more information today than ever in history. Thanks to data collection programs, software and applications for data entry, it is an enormous amount of data that has the potential to provide a revolutionary perspective on how to improve the efficiency of the business model. As a result, business leaders can reap a significant return on investment through detailed analysis of these data. Unfortunately, Big Data is not understood in its true potential, how the supply chain and help you would need profited as soon as possible.

Big Data offers insight to realize reduced costs in the supply chain and a more strategic approach to transport management. Analysis of data collected provides a chance to see how the fare and the route can be optimized and consolidated to improve efficiency and product flow.

World Economic Forum states within the Global Risk Report 2017 [1] that: "technology, in particular, may be where we can turn for innovative solutions to today's 
risks and challenges. It may also produce additional risks which must be assessed and factored in to thinking at business, government, and international levels. New and sometimes revolutionary structures such as Artificial Intelligence systems represent such opportunities for streamlining our everyday lives, but also bring with them many potential dangers. These include mismanagement, design vulnerabilities, accidents, unforeseen occurrences and malicious use that post risks to security and safety of individuals."

Safety and sustainability is a priority for carriers [8]. A safety problem could lead to negative reactions with rapid financial failures. Therefore, automated systems can help increase safety by eliminating the possibility of human error from the equation. In other words, transport will be organized to prevent accidents at work by using automated systems. There was a time when logistics was composed of simple transport processes. But modern logistics is data-driven, and this concept will become essential for success

\section{References}

1. $* * *$ http://www3.weforum.org/docs/GRR17_Report_web.pdf, Accessed in March 2017

2. $\quad * * *$ http://www.engr.washington.edu/news/trend, Accessed in March 2017

3. *** http://www.un.org/ga/search/view_doc.asp?symbol=A/RES/70/1\&Lang=E, Accessed in March 2017

4. ***http://www.oxfordmartin.ox.ac.uk/downloads/commission/Oxford_Martin_Now_for_the_Lo ng_Term.pdf, Accessed in March 2017

5. D. Badea, and S.M. Petrişor, Proceedings of the $16^{\text {th }}$ International Conference The Knowledge Based Organisation, Sibiu, 479-484 (2010)

6. S. Singh, New Mega Trends: Implications for our Future Lives, (Palgrave Macmillan, 2012)

7. *** http://www.investinbsr.com/ipaforum/wp-content/uploads/Iain-Jawad-IPA-Forum-2014Presentation.pdf, Accessed in March 2017

8. L. Ivascu, M. Tămășilă, I. Tăucean, L.I. Cioca, M. Izvercian, Proceedings of the $5^{\text {th }}$ Annual International Conference on Cognitive-Social, and Behavioural Sciences, 259-267 (2017) 